\title{
A Combined Co-location Pattern Mining Approach for Post-Analyzing Co-location Patterns
}

\author{
Yuan Fang, Lizhen Wang*, Junli Lu and Lihua Zhou \\ Department of Computer Science and Engineering, School of Information Science and Engineering, Yunnan University, Kunming \\ 650091, China \\ ${ }^{*}$ Corresponding author
}

\begin{abstract}
The co-location pattern mining discovers the subsets of spatial features which are located together frequently in geography. However, the huge number of the co-location mining results limit the usability of co-location patterns. Furthermore, users hardly identify and understand the interesting knowledge directly from the single co-location pattern.In this paper, we studied the problem of extractingcombined co-location patterns from a large collectionof prevalent co-location patterns. We first gave the definitions of atomic co-location pattern, combined co-location pattern pair and cluster; secondly, we designed a series of interesting metrics to measure the interestingness of atomic co-location patterns, combined co-location pattern pairs and clusters; thirdly, an combined co-location mining algorithm and redundant elimination strategies were proposed. The experiments evaluated the method both on real data sets and syntheticdata sets. The results show that our method can effectively discover combined co-location patterns.
\end{abstract}

Keywords-co-location pattern mining; combined mining; post-analysis

\section{INTRODUCTION}

Spatial co-location mining has been a focused research theme in spatial data mining due to its broad applications at Earth science, environmental protection, public transportation, public safety, GIS, military systems engineering, and urban planning.

The spatial co-location mining was firstly proposed by [2] aims to discover the subsets of spatial features which are located together frequently in a spatial neighborhood.In next decade, many approaches and algorithms are proposed for improving the co-location mining efficiency[3-7], applying the co-location mining technology in different data types[8-11](eg: spatial uncertain data) and [12-13] introducing the applications to special cases (eg: co-location mining with rare spatial features). Example 1 shows the co-location mining process.

Example 1: Figure I shows an example of spatial data set. The instances of features are represented by some points. If instances are neighbors, they are connected with a line.There are 5 spatial features $F=\{\mathrm{A}, \mathrm{B}, \mathrm{C}, \mathrm{D}, \mathrm{E}\}$, Feature A has 6 instances, $\mathrm{B}$ has 5 instances, $\mathrm{C}$ has 5 instances, $\mathrm{D}$ has 4 instances and $\mathrm{E}$ has 3 instances.

For the co-location pattern $c=\{\mathrm{A}, \mathrm{B}\}$, the table instance of $c P R(c, \mathrm{~A})=5 / 6, P R(c, \mathrm{~B})=4 / 5, P I(c)=\min (P R(c, \mathrm{~A}), P R(c, \mathrm{~B}))=$ $0.8 \geq 0.33$. The mining results show in Figure I.

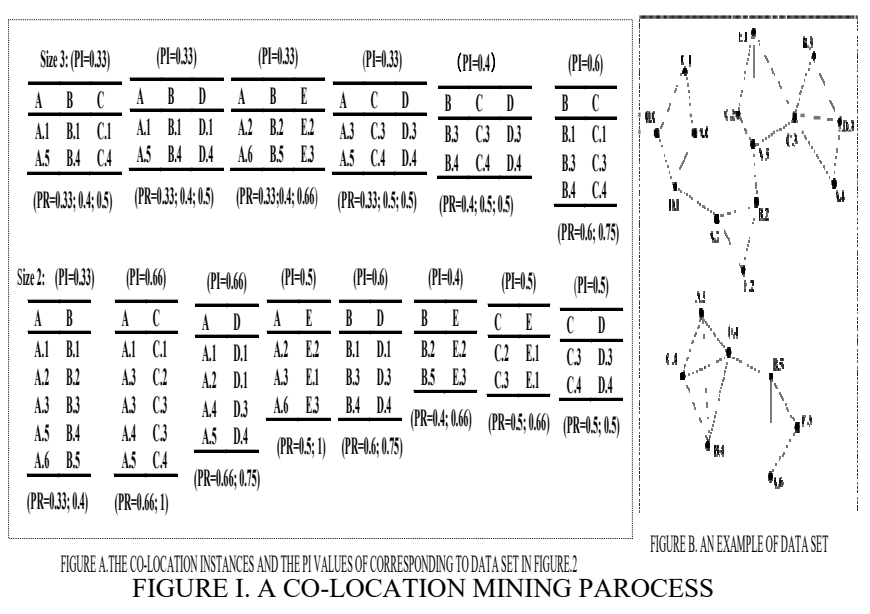

The co-location mining process usually produces large collections of results. In addition, most of co-location mining algorithms adopt a level-wised search method (Apriority-like) whichgenerates numerous redundant patterns.Because of the huge numbers of prevalent co-location patterns, users hardly identify the direct and comprehensive information they interestedefficiently.

Conventional co-location pattern miningfocuses on discovering the relationship in spatial features, it provides limited information for the relation in co-location patterns. In some cases, single prevalent co-location pattern may offer partial even incorrect information to users. For example, there are 5 spatial features:Restaurant, Hospital,Supermarket, School and Movie Theater.The co-location pattern mining produces 20 prevalent co-location patterns such as \{restaurant, hospital\}. A user wants to set some vending machines in public places like schools and hospitals, and the vending machines must keep away fromthe supermarkets. Unfortunately, the user may feel difficult to identify the direct information from the vast results and extract useful knowledge to support decisions.In this situation, combine the co-location patterns and make further analysis to the patterns arenecessary.

Combined co-location patterns are a special case of combined patterns[14-16]. Firstly, the combined co-location pattern mining is focused on the spatial prevalent co-location patterns, the differences of our work are no concepts of transactional itemsets and no contingency table in spatial database. Secondly, the conventional transactional itemsets are dependent, spatial features are correlative, the combined method of them are different. Thirdly, analyzing spatial feature 
relations and co-location pattern relations are more complicated than transaction object relations and pattern relations for additionally considering spatial data distribution. Based on above, we open up a new prospect to detect the combined co-location pattern mining among multi-features in spatial databases.

The major challenge of co-location post-analysis is not at reducing the scale of the spatial co-location mining result set, but at extracting comprehensiveand interesting information. To solve the problem, we extend the conventional combined mining technique [1] to spatial co-location mining. Through combining the co-location patterns to some groupsbased on user preference, measuring their interestingness and extracting the comprehensive information, we can discover and analyze the relationship of colocation patterns. The comprehensive and interesting information corresponding to a user preference can be provided directly.

This paper proposes an approach to mine combined co-location patterns, which is based on prevalent co-locations. The main contributions of our work are 1) a series of definitionsreferred to combined co-location patterns, including atomic co-location patterns, combined co-location pairs and combined co-location clusters. 2)A series of interestingness metricsare designed for measuring the combined co-location patterns.3)Redundancy eliminating strategy is proposed. 4) An efficient algorithm for combined pattern mining; and the experiment results show theperformance of proposed approach.

The remainder of the paper is organized as follows: Section 2 describes related definitions and proposes interestingness measures; Section 3 presents a combined co-location mining algorithm, and the experimental evaluation is presented in Section 4; Section 5 ends this paper with the summary and the future works.

\section{Problem StATEMENT}

In this section, we firstly give the definition of atomic co-location pattern, then introduce a series related definitions to formulate the combined co-location pattern extraction problem.

\section{A. Definition of Combined Co-location Patterns}

Compared with conventional transaction data mining, spatial data is correlated. That means during the combined co-location pattern mining process, if all features are treated equally, we have to consider all the feature combinations for each prevalent co-location pattern, the computation complexity will be NP-Hard.

In this situation, we introduce user preferencesthat allow users choose the features they interested.In this paper, according to users' interests, we divide the features set into two sets:interesting set and the reference set.Interesting set stands for features which are user choose,reference set consistsof the features which not in the interesting set. Each co-location pattern in prevalent co-location pattern set consists of interesting features which belong tothe interesting feature set and reference features belong to the reference feature set.

Definition 1 (Atomic Co-location Pattern)
Given a feature set $F$, an interesting feature $\operatorname{set} F \mathbf{I}(F I \subseteq F)$, a reference feature $\operatorname{set} F R(F R=F \mid F I)$, a $k$-size prevalent co-location pattern $c=\left\{f_{1}, f_{2}, \ldots, f_{k}\right\}, c$ is divided into $I$ set and $R$ set: $c: I \cup R$, where $I \subseteq F I, R \subseteq F R, I \neq \emptyset, R \neq \emptyset, I \cap R=\emptyset$

$I \cup R$ is defined as an atomic co-location pattern, atomic co-location pattern is basic unit of combined co-location patterns. Note that if a co-location pattern cannot be divided intoset $I$ and $\operatorname{set} R$, it is not an atomic co-location pattern.

The distribution of spatial data is correlated, that means the co-location patterns which share same features may correlated. For analyzing the relation of the co-location patterns, we combine the atomic co-location patternsinto pairs/clusters. In combination process, we hope that the set $I$ shares enough similarity and the set $R$ is distinct to provide enough contrast and comprehensive information.

\section{Definition 2 (Combined Co-location Pattern Pair)}

Given two atomic co-location patterns $I \cup R_{1}, I \cup R_{2}, R_{1}$ and $R_{2}$ are different subsets of $F R$, a combined co-location pattern pair $\mathrm{P}$ is defined as $P:\left\{\begin{array}{l}P_{1}: I \cup R_{1} \\ P_{2}: I \cup R_{2}\end{array}\right.$, where $I \neq \emptyset, R_{1} \neq \emptyset, R_{2} \neq \emptyset, R_{1} \cap R_{2}=\emptyset$

\section{Definition 3( Combined Co-location Pattern Cluster)}

A combined co-location pattern cluster is a set of atomic co-location patterns which share the same $I$ but different $R$. A combined co-location pattern cluster $C$ is defined as $C:\left\{\begin{array}{l}P_{1}: I \cup R_{1} \\ P_{2}: I \cup R_{2} \\ P_{n}: I \cup R_{n}\end{array}\right.$

where $I \neq \emptyset, R_{1} \neq \emptyset, R_{2} \neq \emptyset, \ldots, R_{n} \neq \varnothing$

Note that the combined co-location pattern cluster is formed by a most interesting combined co-location pattern pairs with other atomic combined co-location patterns which share same $I$ set.Next we introduce the interesting metrics of combined co-location pairs and clusters.

\section{B. Interestingness Measures}

For the peculiarity of the co-location mining, traditional interestingness is not appropriate for measuring combined co-location pattern mining. New interestingness metrics are designed as follows for measuring the interestingness of atomic co-location patterns, combined co-location pattern pairs and clusters.

In this paper, we use the average participation ratio ofthe features in $I(R)$ set for measuring $I(R)$ contributes to atomic co-location pattern.

\section{Definition4(Interestingness of atomic co-locationPattern)}

Given an atomic co-location pattern $I \cup R, k_{i}$ is the size of the $\operatorname{set} I(I \subseteq F I)$, and $k_{r}$ is the size of the $\operatorname{set} R(R \subseteq F R)$. The interestingness metric of $I \cup R$ measures the contribution of $I$ or $R$ to $I \cup R$ respectively. The interestingness of $I$ to $I \cup R$ is designed as follows:

$$
\mathrm{IA}_{I}(I \cup R)=\frac{1}{|I|} \sum_{i=1}^{k_{i}} \frac{\mid \pi_{f}(\text { table_instance }(\{I \cup R\})) \mid}{\mid \text { table_instance }\left(\left\{f_{i}\right\}\right) \mid}
$$


$\mathrm{IA}_{I}(I \cup R)$ is the average participation ratio of all features of $/$ in $I \cup R$. Similarly, we extend our formulation to the interestingness of $R$ to $I \cup R$.

$$
\operatorname{IAR}(I \cup R)=\frac{1}{|R|} \sum_{j=1}^{k_{\mathrm{r}}} \frac{\mid \pi_{\mathrm{f}_{\mathrm{j}}}\left(\operatorname{table} \_ \text {instance }(\{\mathrm{I} \cup \mathrm{R}\})\right) \mid}{\mid \text { table_instance }\left(\left\{\mathrm{f}_{\mathrm{j}}\right\}\right) \mid}
$$

Note that theset $R$ represents the reference part of $I \cup R$, the more contribution of $R$ to $I \cup R$, means $R$ has more power to affect $I$.

Considering the correlation of co-location patterns, if two co-location patterns share same features, and their instances of these features are coincident. The more correlated of two atomic co-location patterns, the less contrast of these two co-location patterns. We designed a metric for measuring the correlation of two atomic co-location patterns.

\section{Definition5(Co-location_Coincident_Ratio)}

Given two atomic co-location patterns $I \cup R_{1}, I \cup R_{2}$, which contain same $I$ but different $R$, Co-location_Coincident_RatioCCR $\left(\left\{I \cup R_{1}\right\},\left\{I \cup R_{2}\right\}\right)$ is defined as follows:

$\max _{\mathrm{i}=1} \mathrm{k}_{\mathrm{i}}\left(\frac{\mid \pi_{\mathrm{f}}\left(\text { table_instance }\left(\left\{\mathrm{I} \cup \mathrm{R}_{1}\right\}\right)\right) \cap \pi_{\mathrm{f}}\left(\operatorname{table} \_ \text {instance }\left(\left\{\mathrm{I} \cup \mathrm{R}_{2}\right\}\right)\right) \mid}{\max \left\{\mid \pi_{\mathrm{f}_{\mathrm{i}}}\left(\operatorname{table} \_ \text {instance }\left(\left\{\mathrm{I} \cup \mathrm{R}_{1}\right\}\right)\right)|,| \pi_{\mathrm{f}_{\mathrm{i}}}\left(\operatorname{table} \_ \text {instance }\left(\left\{\mathrm{I} \cup \mathrm{R}_{2}\right\}\right)\right) \mid\right\}}\right)$

CCR contributes the correlation of spatial features, the higher CCR means the more same instances of $I$ existsin both $I \cup R_{l}$ and $I \cup R_{2}$, i.e. the two atomic co-location patterns $I \cup R_{l}$ and $I \cup R_{2}$ share enough similarity of $I$, combined them is meaningless without enough contrast.Considering the similarity of $I$ and the distinction of $R$, the interestingness of a combined co-location pattern pair is given in next definition.

Definition6(Interestingness of combined co-location pattern $\operatorname{pairs}(I C P))$

Given a combined co-location pattern pair $C P\left\{\begin{array}{l}C P_{1}: I \cup R_{1} \\ C P_{2}: I \cup R_{2}\end{array}\right.$, the interestingness of $\mathrm{CP}$ is defined as

$\operatorname{ICP}(C P)=\operatorname{IA}_{R}\left(I \cup R_{l}\right) \operatorname{IA}_{R}\left(I \cup R_{2}\right)\left(1-\mathrm{CCR}\left(\left\{I \cup R_{l}\right\},\left\{I \cup R_{2}\right\}\right)\right)$

In this paper, $\operatorname{ICP}(C P)$ is also written as $\operatorname{ICP}\left(C P_{1}, C P_{2}\right)$. ICPmeasures the contribution of the two different reference feature sets to the same interesting feature set. The higher ICP is, the more interesting a combined co-location pattern pair.

Considering set $I$ need enough similarity in the combined co-location pattern clusters, in this paper, we choose the atomic pattern which has maximumI $A_{I}$ to be the cluster center. The interestingness of a co-location pattern cluster is the maximum interestingness of pair which consists of the cluster center pattern and the other atomic co-location pattern in the cluster.

Definition 7(Interestingness of combined co-location pattern cluster)

Given acombined co-location patterncluster CC composed of $n$ atomic co-location patterns $I \cup R_{l}, I \cup R_{2}, \ldots I \cup R_{n},\left\{I \cup R_{c}\right\}$ is the cluster center which with maximum $\operatorname{IA}_{R}\left(I \cup R_{c}\right)$ of all the atomic co-location patterns in the cluster,the interestingness of combined co-location pattern $C C:\left\{\begin{array}{l}C P_{1}: I \cup R_{1} \\ C P_{2}: I \cup R_{2} \\ \ldots P_{n}: I \cup R_{n}\end{array}\right.$ is defined as follows:

$$
\operatorname{ICC}(C C)=\max _{j \in n-1} \operatorname{ICP}\left(I \cup R_{c}, I \cup R_{j}\right)
$$

The $\left\{I \cup R_{c}\right\}$ is the cluster center, we choose the maximum interestingness combined co-location pairs consist of $\left\{I \cup R_{c}\right\}$ with other $n-1$ atomic co-location patterns. The interestingness metrics of the combined co-location pattern clusters is the maximum interestingness of combined co-location pattern pairs $\left\{I \cup R_{c}, I \cup R_{j}\right\}$.Users can extract the most interesting combined co-location patternpairs, meanwhile, other atomic combined co-location patterns in the cluster can provide additional information.

The combined co-location mining results of Figure I are show in table I.

\section{Eliminating Redundancy Strategy}

In combined pattern mining process, there are 6 types of redundancy for the correlation co-location patterns. The Redundancy Strategy will be shown in algorithm.

TABLE I. THE INTERESTINGNESS OF COMBINED CO-LOCATION CLUSTER AND

\begin{tabular}{|c|c|c|c|c|c|c|}
\hline cluster & $\begin{array}{l}\text { Atomic } \\
\text { Pattern }\end{array}$ & $I A_{R}$ & $I A_{I}$ & CCR & ICP & ICC \\
\hline \multirow[t]{4}{*}{$\mathrm{C} 1$} & $\{A\} \cup\{C\}$ & 0.75 & 0.66 & \multirow{4}{*}{$\begin{array}{l}P 1:\{A\} \cup\{C\},\{A\} \cup\{D\}: 0.6 \\
P 2:\{A\} \cup\{C\},\{A\} \cup\{E\}: 0.2 \\
P 3:\{A\} \cup\{C\},\{A\} \cup\{C, D\}: 0.4 \\
P 4:\{A\} \cup\{D\},\{A\} \cup\{E\}: 0.25 \\
P 5:\{A\} \cup\{D\},\{A\} \cup\{C, D\}: 0.5 \\
P 6:\{A\} \cup\{E\},\{A\} \cup\{C, D\}: 0\end{array}$} & \multirow{4}{*}{$\begin{array}{l}\text { P1:0.23 } \\
\text { P2:0.6 } \\
\text { P3 } \\
\text { P4:0.56 } \\
\text { P5 } \\
\text { P6:0.5 }\end{array}$} & \multirow{4}{*}{0.6} \\
\hline & $\{\mathrm{A}\} \cup\{\mathrm{D}\}$ & 0.75 & 0.66 & & & \\
\hline & $\{A\} \cup\{E\}$ & 1 & 0.5 & & & \\
\hline & $\{\mathrm{A}\} \cup\{\mathrm{C}, \mathrm{D}\}$ & 0.5 & 0.33 & & & \\
\hline \multirow[t]{4}{*}{$\mathrm{C} 2$} & $\{\mathrm{~B}\} \cup\{\mathrm{C}\}$ & 0.75 & 0.6 & \multirow{4}{*}{$\begin{array}{l}P 1:\{B\} \cup\{C\},\{B\} \cup\{D\}: 1 \\
P 2:\{B\} \cup\{E\},\{B\} \cup\{C, D\}: 0\end{array}$} & \multirow[t]{4}{*}{ P2:0.33 } & \multirow[t]{4}{*}{0.33} \\
\hline & (B) $\cup[\mathrm{D}\}$ & 0.75 & 0.6 & & & \\
\hline & $\{B\} \cup\{E\}$ & 0.66 & 0.4 & & & \\
\hline & $\{\mathrm{B}\} \cup\{\mathrm{C}, \mathrm{D}\}$ & 0.5 & 0.4 & & & \\
\hline \multirow[t]{3}{*}{$\mathrm{C} 3$} & $\{A, B\} \cup\{C\}$ & 0.5 & 0.37 & \multirow{3}{*}{$\begin{array}{l}P 1:\{A, B\} \cup\{C\},\{A, B\} \cup\{D\}: 1 \\
P 2:\{A B\} \cup\{D\},\{A B\} \cup\{E\}): 0\end{array}$} & \multirow[t]{3}{*}{ P2:0.25 } & \multirow[t]{3}{*}{0.25} \\
\hline & $\{A, B\} \cup\left\{D_{\}}\right\}$ & 0.5 & 0.37 & & & \\
\hline & $\{\mathrm{A}, \mathrm{B}\} \cup\{\mathrm{E}\}$ & 0.5 & 0.37 & & & \\
\hline
\end{tabular}
REDUNDANCY ELIMINATION

\section{ALGORITHMS}

In this section,we will discuss a general algorithm for mining combined co-location patterns on prevalent co-location patterns.

\footnotetext{
Algorithm

Input: $C P$ :A collection of prevalent co-locations and their table instances, FI : a user specified interesting feature set

Output: A collection of combined co-location patterns :CCP

Variables : AP: A collection of atomic combined co-location pattern

I: the interesting part of a co-location pattern $(I \subseteq F I)$

$R$ : the reference part of a co-location pattern $(R \subseteq F \mid F I)$

Method:

BEGIN
} 


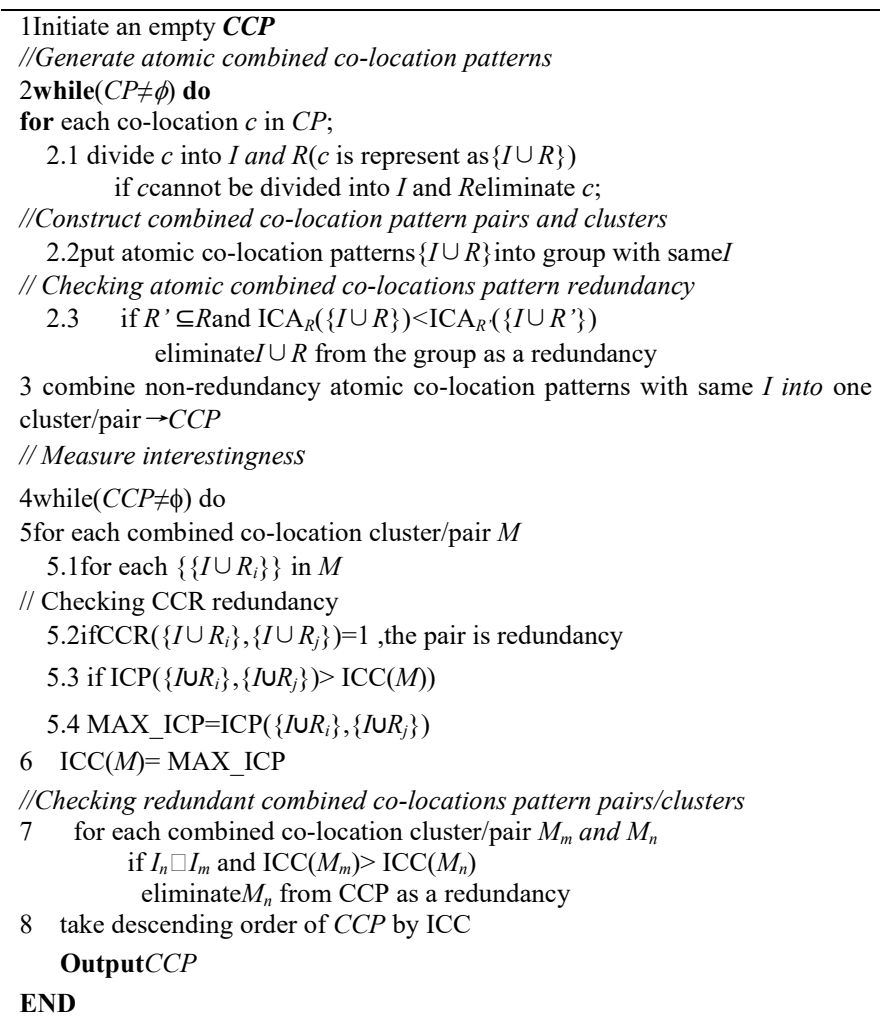

The algorithm contains 3 steps.

In first step, According to the user specified interesting feature set $F I$ and the reference feature $\operatorname{set} F R=\{F \mid F I\}$, dividing each co-location pattern into interesting feature set $I(I \subseteq F I)$ and reference feature $\operatorname{set} R(R \subseteq F R)$, eliminate the co-location patterns which cannot divided into $I$ and $R$.When inserting a atomic co-location pattern $c=\{I \cup R\}$ into $\mathrm{AP}$, compute $\mathrm{IA}_{R}(\mathrm{I} \cup$ $\mathrm{R})$ and check redundancy. $\left.\operatorname{IfIA}_{R}(\{I \cup R\})<\mathrm{IA}_{R}\left(\left\{I \cup R^{\prime}\right\}\right)\right)$ and $R^{\prime} \subseteq R, I \cup R$ is the redundant atomic combined co-location pattern. For example, for $\{\mathrm{A}\} \cup\{\mathrm{C}\},\{\mathrm{A}\} \cup\{\mathrm{C}, \mathrm{D}\}$, if $\mathrm{IA}_{c}(\{\mathrm{~A}\}$ $\cup\{\mathrm{C}\})<\mathrm{IA}_{\mathrm{CD}}(\{\mathrm{A}\} \cup\{\mathrm{C}, \mathrm{D}\}$, then $\{\mathrm{A}\} \cup\{\mathrm{C}\}$ is a redundant atomic co-location pattern.

The second step, we combined atomic co-location patterns. If atomic co-location patterns have the same $I$ set, they are organized in a pair or cluster. Through computing the CCR of each pair $c p$, if $\operatorname{CCR}(c p)=1$, we can eliminate it firstly which impossible to be an interesting pair. The rest of pairs and clusters are put into a combined co-location pattern set CCP(in table I).

The last step we measure the interestingness of combined co-location pattern pairs and clusters. The combined co-location pattern pairsare eliminated though comparing set/between two combined co-location pairs and their ICP (in table I).

\section{PERFORMANCE STUdY}

In this section, due to the related research of combined co-location pattern mining has not been reported, we compared our proposed algorithm with a general co-location mining algorithm: Join-less Approach[3].We use two kinds of datasets in our experiments: real datasets and a series of synthetic datasets. All the algorithms are implemented in Visual C\#. All of ourexperiments are performed on a $2.20 \mathrm{GHZ}$, 4GB-memory, Inter PC running Windows 7.

\section{A. Data Sets}

For evaluating the algorithm performance, we use two kinds of datasets in our experiments: real dataset and synthetic datasets. The real dataset are points of interesting of Beijing?,this dataset consist of51,546 spatial instances with 20 distinct features.

TABLE II. A DESCRIPTION OF DATA SETS USED IN EXPERIMENTS

\begin{tabular}{|c|c|c|c|}
\hline dataset & Instance Amount & Feature amount & Data type \\
\hline Dataset1 & 20,000 & 20 & synthetic \\
\hline Dataset2 & 30,000 & 20 & synthetic \\
\hline Dataset3 & 50,000 & 25 & synthetic \\
\hline Dataset4 & 80,000 & 28 & synthetic \\
\hline Dataset5 & 51,546 & 20 & real \\
\hline
\end{tabular}

B. The Mining Results of Combined Mining vs General Co-Location Pattern Mining

We compare the combined co-location clusters discovered byour method (called CCC in the following) with the results mined by the general co-location mining approach:Join-less algorithm.Figure II shows the number of the Join-less mining results and the number of combined co-location clusters and pairs discovered by $\mathrm{CCC}$ over the prevalent co-location patterns. The experiment is running with different participation index threshold min previn Dataset 1, the neighbor relation distance threshold is 20 , the interesting feature amount is 10 in CCC algorithm.

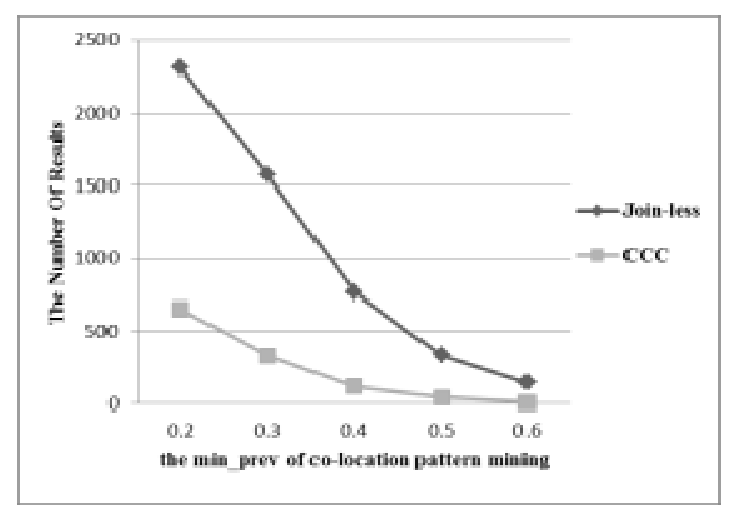

FIGURE II THE COMPARE OF MININGRESULTS

Figure II indicates that $\mathrm{CCC}$ algorithm can reduce the number of conventional co-location pattern results effectively.

\section{Efficiency}

To compare the time efficiency of CCC and Join-less,our experiments test the running time of $\mathrm{CCC}$ algorithm with increasing data size is shown in Table II. Figure III shows the time efficiency of Join-less algorithm and CCC algorithm in increasing size of datasets. The participation index threshold min prev is 0.4 , the neighbor relation distance threshold is 20 , the interesting feature amount is 10 in $\mathrm{CCC}$ algorithm. 


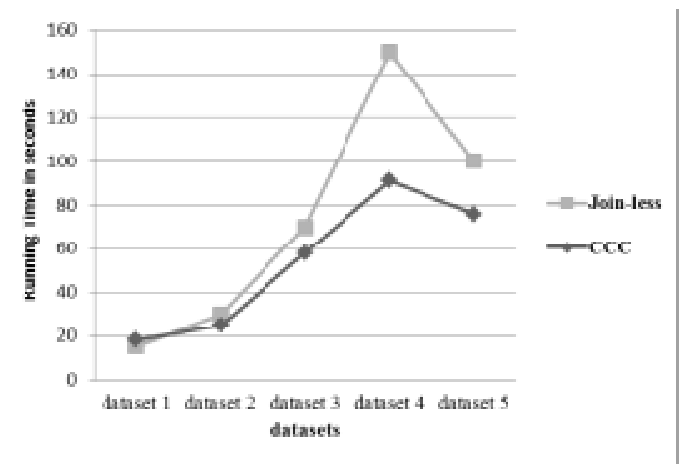

FIGURE III. RUNNING TIME FIGURE

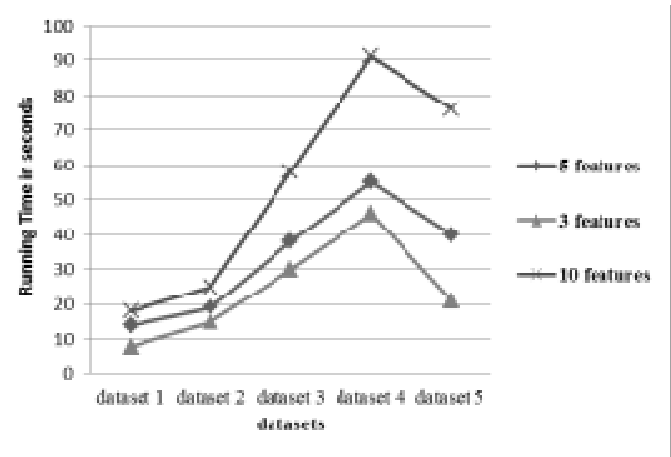

FIGURE IV. RUNNING TIME WITH DIFFERENT DATASETSWITH INCREASING FEATURES

The experiment in Figure III shows that the running time of $\mathrm{CCC}$ is stable increasing in different size of datasets.

Totest the effect of the interesting feature amount to efficiency of CCC with increasing interesting features, our experiments test the execution time of $\mathrm{CCC}$ algorithm with increasing data size shown in Table I. We test the execution timeof CCC in 3 interesting features,5interesting features and 10 interesting features in Figure IV.

The experimental results in Figure IV shows that the running time of $\mathrm{CCC}$ is increasing by increasing features, the major expense of CCC algorithm is compute coincident instances of interesting features, the running time of CCC is increase rapidlyby increasing feature amount.

\section{The Real Applications of Combined Co-location Mining}

We use real data sets to analyze the applications of combined co-location mining. Table II is the results of combined co-location mining, the mining results shows that the combined co-location pattern clusters/pairs can offer comprehensive and direct information.

In some cases, potential information is hidden in large numbers of co-location pattern mining results. The number of combined co-location pattern clusters/pairs is significantly smaller than prevalent co-location patterns, it is available to usersobtain potential information which can not know from single co-location pattern. Table III shows the 3 interesting combined co-location pattern clusters and their interestingness. In the cluster $\mathrm{C} 1$ we can find the three co-location patterns are related with school, the high ICC means the table instances of these patterns are low coincidence. We can analyze that the school type in these patterns are different. Generally, the nursery schools are close to residential area, universities are close to ATM, and many adult trainingschools are close to CBD. Cluster C3 also shows the different types of supermarkets are always close to distinct areas. In cluster $\mathrm{C} 2$ we can find that parking lots are always close to toilet, however, when parking lots are located with CBD, there are notoilet located with them, the information may helpful to public service.

TABLE III. THE RESULTS OF COMBINED CO-LOCATION MINING

\begin{tabular}{|l|l|l|}
\hline \multicolumn{2}{|l|}{ Combined co-location pattern cluster } & ICC \\
\hline C1 & $\begin{array}{l}\text { \{school,residential area\} } \\
\text { \{school, ATM } \\
\text { \{school, CBD }\end{array}$ & 0.356 \\
\hline C2 & $\begin{array}{l}\text { \{parking lots, Public toilet, Scenic\} } \\
\text { \{ Parking lots, CBD } \\
\text { \{parking lots, Public toilet, Crossroad }\end{array}$ & 0.29 \\
\hline C3 & $\begin{array}{l}\text { \{supermarket,hotel }\} \\
\text { ssupermarket,residential area } \\
\text { ssupermarket,CBD }\end{array}$ & 0.22 \\
\hline
\end{tabular}

Single co-location pattern cannot provide comprehensive information, In fact, the majority of co-location patterns can not represent all adjacent relationship which related to their own. We can find some potential and interesting information through combining co-locations.

\section{CONCLUSIONS AND FUTURE WORKS}

In this paper, we studied the combined co-location patterns over prevalent co-locations. We adopt the concept of combined mining to presented a framework for efficiently mining the combined co-location patterns from prevalent co-location setto reduce the co-location mining resultsand provide direct, comprehensive knowledge.We proposea general combined co-location mining method and redundancyelimination strategies. Furthermore, the combined co-location mining algorithm is presentedand the experiments on real datasets and synthetic datasets indicate that our algorithm can reduce the co-location mining results and give the comprehensive information. As a future work, we plan to extend combined co-location mining to other different types of data.

\section{ACKNOWLEDGMENT}

This work was supported in part by grants (No. 61472346,No.61262069) from the National Natural Science Foundation of China, in part by a grant (No. 2015FB149, No.2015FB114) from the Science Foundation of Yunnan Province and in part by the Seventh of Yunnan University Graduate Student Scientific Research Project under Grant No.YNUY201444.

\section{REFERENCES}

[1] L. Cao, "Combined mining: Analyzing object and pattern relations for discovering and constructing complex yet actionable patterns,"Wiley Interdisciplinary Reviews: Data Mining and Knowledge Discovery. vol.3,pp. 140-155, 2013.

[2] Y. Huang, S. Shekhar, H.Xiong, "Discovering Co-Location Patterns from Spatial Data Sets: A General Approach," IEEE Transactions on Knowledge and Data Engineering (TKDE).vol.12,pp. 1472-1485,2004. 
[3] J. S. Yoo and S. Shekhar, "A joinless approach for mining spatial co-location patterns," IEEE Transactions on Knowledgeand Data Engineering (TKDE), vol. 10,pp. 1323-1337,2006.

[4] J. S. Yoo, S. Shekhar, J. Smith, and J. P. Kumquat, "A partial join approach for mining co-location patterns," Proc. 12th annual ACM International Workshop on Geographic Information Systems (GIS), pp.241-249,2004.

[5] J. S. Yoo and M. Bow, "Mining Top-k Closed Co-Location Patterns,"In Proceedings of IEEE International Conference on Spatial Data Mining and Geographical Knowledge Services (ICSDM), pp.100-105,2011.

[6] L. Wang, Y. Bao, Z.Lu,"Efficient Discovery of Spatial Co-Location Patterns Using the iCPI-tree,"The Open Information Systems Journal, vol.3,pp. 69-80, 2009.

[7] J. S. Yoo and M. Bow,"Mining Maximal Co-Located Event sets," In: Proceedings of Pacific-Asia International Conference on Knowledge Discovery and Data Mining (PAKDD 2011), Lecture Notes in Computer Science, Vol. 6634,pp. 351-362, 2011.

[8] L. Wang, Y. Bao, J. Lu, and J. Yip, "A new join-less approach for co-location pattern mining," Proc. 8th IEEE International Conference on Computer and Information Technology (CIT),pp.197--202,2008.

[9] L. Wang, H. Chen, L.Zhao and L.Zhou, "Efficiently Mining Co-location Rules on Interval Data,"(ADMA 2010 Part I, LNCS 6440), pp. 477-488,2010.

[10] [10]L. Wang, P. Wu, and H. Chen,"Finding Probabilistic Prevalent Co-Locations in Spatially Uncertain Data Sets,"IEEE Transactions on Knowledge and Data Engineering(TKDE), vol. 4,pp.790-804,2013.

[11] Y. Lu, L. Wang, andX. Zhang, "Mining Frequent Co-location Patterns from Uncertain Data," Journal of Frontiers of Computer Science and Technology, vol. 6,pp. 656-664,2009.

[12] Y. Huang, J. Pei, and H. Xiong, "Mining Co-location Patterns with Rare Events from Spatial Data Sets," GeoInformatica, vol. 3,pp. 239-260,2006.

[13] L. Feng, LZ. Wang, and SJ. Gao, "A new approach of mining co-locaiton patterns in spatial datasets with rare feature,"Journal of Nanjing University(Natural Sciences), vol. 48, pp. 99-107 ,2012.

[14] Y. Zhao, H. Zhang, F. Figueiredo, L. Cao, and C. Zhang,"Mining for combined association rules on multiple datasets," International Conference on Knowledge Discovery and Data Mining, 2007.

[15] Y. Zhao, H. Zhang, L. Cao, C. Zhang, and H. Bohlscheid."Combined Pattern Mining: From Learned Rules to Actionable Knowledge,” Lecture Notes in Computer Science, Vol.5360, pp. 393-403,2008.

[16] L. Cao, "Actionable knowledge discovery and delivery," Wiley Interdisciplinary Reviews: Data Mining and Knowledge Discovery,vol.2,pp.149-163.2012. 